\title{
BMJ Open Quality of life and objective functional impairment in lumbar spinal stenosis: a protocol for a systematic review and meta-analysis of moderators
}

\author{
Fabio Ferretti, ${ }^{1}$ Anna Coluccia, ${ }^{1}$ Roberto Gusinu, ${ }^{2}$ Giacomo Gualtieri, ${ }^{3}$ \\ Vitaliano Francesco Muzii (D) , ${ }^{1}$ Andrea Pozza (i) ${ }^{1}$
}

To cite: Ferretti F, Coluccia A, Gusinu R, et al. Quality of life and objective functional impairment in lumbar spinal stenosis: a protocol for a systematic review and meta-analysis of moderators. BMJ Open 2019;9:e032314. doi:10.1136/ bmjopen-2019-032314

- Prepublication history and additional material for this paper are available online. To view these files, please visit the journal online (http://dx.doi org/10.1136/bmjopen-2019032314).

Received 12 June 2019 Revised 19 September 2019 Accepted 11 October 2019

D) Check for updates

C Author(s) (or their employer(s)) 2019. Re-use permitted under CC BY-NC. No commercial re-use. See rights and permissions. Published by BMJ.

${ }^{1}$ Department of Medical Sciences, Surgery and Neurosciences, Santa Maria alle Scotte University Hospital of Siena, Siena, Italy

${ }^{2}$ Health Service Management Board, Santa Maria alle Scotte University Hospital, Siena, Italy ${ }^{3}$ Legal Medicine Unit, Santa Maria alle Scotte University Hospital, Siena, Italy

Correspondence to Dr Andrea Pozza; andreapozza7@gmail.com

\section{ABSTRACT}

Background Lumbar spinal stenosis (LSS) is a common degenerative spine disease associated with a strong impairment in various quality of life areas, particularly the ability to perform work-related activity. Depression is a condition frequently associated. There is no comprehensive review on quality of life and objective functional impairment in LSS. This paper presents the protocol of the first systematic review and meta-analysis summarising evidence about quality of life and functional impairment in patients with LSS compared with healthy controls. Comorbid depressive disorders, age, gender, LSS duration, disability, pain severity and study methodological quality will be investigated as moderators.

Methods The protocol is reported according to PRISMA-P guidelines. Studies will be included if they were conducted on patients aged 18 years old or older with primary LSS and if they reported data on differences in the levels of quality of life or objective functional impairment between patients with LSS and healthy controls. Independent reviewers will search published/unpublished studies through electronic databases and additional sources, will extract the data and assess the methodological quality. Random-effects meta-analysis will be carried out by calculating effect sizes as Cohen's $d$ indices. Heterogeneity will be examined by the $R$ and the $Q$ statistics. Moderators will be investigated through meta-regression. Conclusions A summary of the evidence on quality of life and functional impairment in LSS may suggest clinical and occupational health medicine strategies aimed to timely detect and prevent these outcomes. Higher percentages of patients with LSS with depression may be expected to be related to poorer quality of life. Depressive comorbidity might impact negatively on quality of life because it is associated with dysfunctional coping, disability and psychophysiological symptoms.

Ethics and dissemination The current review does not require ethics approval. The results will be disseminated through publications in peer-reviewed journals.

Review registration CRD42019132209.

\section{INTRODUCTION}

Health-related quality of life: a relevant outcome in lumbar spinal stenosis

Health-related quality of life can be defined as the perceived health status on the ability
Strengths and limitations of the study

- Study selection and data extraction will be performed by independent reviewers.

- If heterogeneity is found, subgroup analyses will be conducted for studies using only clinician-administered interviews or self-report questionnaires.

- Another strength will be the moderator analysis of comorbid depressive disorders based on international standardised classification systems.

- Potential limitations concern a small number of studies and heterogeneity of instruments assessing quality of life.

- Another limitation might be that some studies do report data for moderator coding or the authors do not provide them on request.

to lead a fulfilling daily life. ${ }^{1-11}$ Lumbar spinal stenosis (LSS) is a condition associated with the natural process of ageing leading to narrowing of the lumbar spinal canal and foramen, resulting from a degenerative process. When stenosis is clinically relevant, it results in a syndrome known as neurogenic claudication. Patients generally experience and report activity-related low-back and leg pain that worsens with prolonged standing or ambulation, limiting their walking distance and impacting their capacity to live a fulfilling life. LSS is relatively common among the elderly, affecting more than 200000 adults in the USA, and it is the most frequent reason for spinal surgery in patients over 65 years. ${ }^{12} 13$ As a degenerative spine disease, its prevalence is expected to increase with the continued ageing of the population. ${ }^{14}$ Symptoms are often chronic, frequently missed or misdiagnosed, leading to strong impairment or reduction in quality of life. ${ }^{14}{ }^{15}$ One of the most impaired domains in the patient's life is the ability to perform work-related activity. ${ }^{15}$ 
The clinical picture of LSS is characterised by a diminished space available for the neural and vascular elements in the lumbar spine secondary to degenerative changes in the spinal canal. ${ }^{1617}$ When symptomatic, this causes a variable clinical syndrome of gluteal and/or lower extremity pain and/or fatigue that may occur with or without back pain. ${ }^{17}$ Symptomatology of LSS consists of specific provocative and palliative features. Provocative features include upright exercise such as walking or positionally induced neurogenic claudication. Palliative features typically involve symptomatic relief with forward flexion, sitting and/or recumbency. ${ }^{1617}$

The presence of a narrowed spinal canal on radiographic imaging is not a sufficient criterion to diagnose LSS, and a correlation between narrowing of the spinal canal and clinical symptoms of spinal stenosis has not been demonstrated yet. ${ }^{12} 1417$ Therefore, LSS is mainly a clinical diagnosis supported by consistent radiological findings. ${ }^{17}$

The diagnosis of LSS may be considered in older patients presenting with neurogenic claudication and imaging studies demonstrating narrowing of the spinal canal. Neurogenic claudication represents the key symptomatic aspect of LSS, defined as intermittent pain radiating to the buttocks, thighs and/or lower legs that is typically provoked by standing, walking and/or lumbar extension, and relieved with sitting, lying down or lumbar flexion. If the level of intensity is severe, neurogenic claudication determines considerable difficulties in walking. ${ }^{18-20}$

In patients with history and physical examination evidence consistent with LSS, MRI is generally considered as the most reliable non-invasive tool aimed to support the presence of anatomical narrowing of the spinal canal or the presence of nerve root impingement. ${ }^{17}$ It can also enhance the differential diagnosis with peripheral neuropathy, lumbar spondylosis and peripheral artery disease, whose symptoms may resemble LSS. ${ }^{16} 1821$

A number of studies demonstrated that health-related quality of life is poorer in patients with LSS compared with healthy individuals without this condition and even compared with patients diagnosed with chronic back pain. ${ }^{22}{ }^{23}$ There is a number of reasons why assessing health-related quality of life in LSS is an important strategy for the management of this condition. Patients with poorer quality of life may be expected to have worse self-management skills of symptoms, to engage less in activities to maintain function, to collaborate with healthcare providers and navigate effectively the healthcare system. ${ }^{24}$ Patients with worse quality of life may believe to a less extent that an active role is important in the management of this condition, have lower optimism and hope, lower self-efficacy and locus of control on health behaviour. $^{25}$

Patients with LSS experience significantly lower job satisfaction than individuals without this condition. ${ }^{26}$ In addition, about $20 \%-40 \%$ of the patients with LSS present clinically significant depressive symptoms. ${ }^{27} 28$
In patients with LSS, comorbid depressive disorders are frequent and higher than in controls; this type of comorbidity is often associated with worse well-being, more severe long-term disability (a combination of symptoms of pain, numbness, weakness and balance issues) and more dysfunctional coping such as lower sense of coherence, lower engagement in physical exercise, more severe pain sensitivity and more catastrophic beliefs about pain..$^{29-32}$

Based on these points, some researchers pointed out the need for integrating the assessment of patientreported quality of life with measures of objective functional impairment since functional status of the patient is less prone to a bias due to psychological health ${ }^{33}$ Objective functional measures such as 'Time Up and Go' test are based on a task to be performed by the patient, which is evaluated using an objective assessment of the patient's performance on that task through a standardised testing protocol (ie, time taken, repetitions) and is rated by an observer and/or machine instead of the patient him/ herself. $^{34}$

\section{Rationale and objectives of the present protocol}

Health-related quality of life and objective functional impairment are important outcomes in the assessment and management of LSS. The assessment of both these aspects during clinical practice may suggest a comprehensive evaluation aimed to improve longterm outcome. For example, patient education and psychological interventions aimed to promote patient's resources have been proven effective for the improvement of both clinical outcomes and quality of life in these patients. $^{3536}$

In the scientific literature, there is no systematic review providing a comprehensive summary of the evidence of health-related quality of life and functional impairment in LSS. The current paper presents the protocol of the first systematic review and meta-analysis aimed at providing a quantitative summary of the levels of health-related quality of life and objective functional impairment in patients with LSS compared with healthy control groups. Comorbidity of depressive disorders will be investigated as a moderator if significant heterogeneity in the effect sizes is found. Comorbidity of depressive disorders might impact negatively on quality of life because depression is associated with worse general well-being, dysfunctional coping strategies, long-term disability and psychophysiological symptoms. On one hand, higher percentages of patients with LSS with comorbid depressive disorders may be expected to be related to poorer levels of quality of life; on the other hand, depressive comorbidity may impact less on objective functional impairment which is less influenced by psychological status. ${ }^{334}$ Other moderators will be examined as potentially impacting negatively on quality of life and functional impairment including age, gender, LSS duration, LSS severity (self-reported disability and pain severity related to LSS) and study methodological quality. 


\section{METHODS}

The planned start and end dates for the study are 1 November and 31 December 2019, respectively. The review protocol is presented according to the guidelines of the PRISMA-Protocol (PRISMA-P). ${ }^{37}$

\section{Eligibility criteria}

In accordance with the PRISMA-P guidelines, the criteria considered for inclusion of studies will be related to (a) participants, (b) outcomes, (c) comparators and (d) design. Studies will be included if (a) they are conducted on adult clinical groups aged 18 years old or older with a primary diagnosis of LSS; (b) they reported quantitative data on differences in the levels of health-related quality of life between a group of patients with LSS and a healthy control group or the authors are willing to provide the necessary data when contacted if such data are missing in the study paper; (c) they used the criteria of neurogenic claudication and/or radicular leg symptoms and confirmatory imaging showing LSS at one or more levels to establish the diagnosis of $\mathrm{LSS}^{38}$; (d) they measured healthrelated quality of life through any validated standardised interview or a validated self-report questionnaire such as the Medical Outcome Survey Short Form-36 (SF-36) ${ }^{39}$ (ie, the psychometric properties such as reliability values are reported in the literature) and/or they assessed objective functional impairment based on a task to be performed by the patient, evaluated using an objective assessment of the patient's performance on that task (ie, time taken, repetitions), rated by an observer and/or machine instead of the patient him/herself through a standardised testing protocol; (e) they used a case-control research design (the study may use any other design if it reports the necessary data to compute effect sizes according to inclusion criterion ' $b$ '). Controls include healthy individuals recruited from the general population/community without LSS; the absence of LSS should be ascertained by a physician through history and physical examination or imaging ruling out lumbar spinal stenosis. Case series will be excluded. Trials on the effects of a treatment will be excluded unless they reported (or the authors are available to provide them on request) data regarding the requested outcomes at baseline (ie, before trial entry). No language restriction will be applied. Studies will be included whether they used inpatients or outpatients. No restriction on publication dates will be used. Studies where patients had any comorbid psychiatric disorders according to any version of the Diagnostic and Statistical Manual of Mental Disorders (eg, DSM-IV-TR) ${ }^{40} 41$ will not be excluded because psychiatric comorbidity can be observed in one in three patients. ${ }^{42}$ If the study assessed the number of patients with comorbid depressive disorders, this comorbidity had to be evaluated by the criteria for a major depressive disorder according to an international standardised diagnostic system such as the Diagnostic and Statistical Manual of Mental Disorders (DSM) or the International Classification of Diseases (ICD).

\begin{tabular}{ll} 
Table 1 Electronic search procedure \\
$\begin{array}{ll}\text { Electronic databases } & \text { Search terms (MeSH and } \\
\text { keywords) }\end{array}$ \\
\hline Scopus & MeSH: \\
PubMed & "Quality of Life", OR "Health- \\
EMBASE & Related Quality of Life" \\
Cochrane Library & Boolean operator and keywords: \\
& OR HRQOL \\
& OR Health-Related Quality of Life \\
& OR Life Quality \\
& AND \\
& MeSH: \\
& "Spinal Stenosis" \\
& Boolean operator and keywords: \\
& OR Constriction, Pathologic \\
& OR Lumbar Vertebrae \\
& OR Spinal Canal \\
& OR Spinal Diseases
\end{tabular}

MeSH, Medical Subject Heading.

\section{Information sources and search procedure}

The search procedure will be conducted on 4 November 2019. Studies will be identified by conducting a systematic search of electronic databases using Medical Subject Headings (MeSH terms) and keywords related to "Health-Related Quality of Life" which will be combined through the Boolean operator "AND" with MeSH terms and keywords related to "Lumbar Spinal Stenosis". MeSH terms were created by using the PubMed MeSH on Demand Tool which allowed us to identify relevant $\mathrm{MeSH}$ terms. The search procedure will be conducted using the databases Scopus, PubMed, EMBASE and the Cochrane Library. An overview of the electronic search strategy is provided in table 1. An example of the search strategy is provided in the Supplementary file. In order to define and validate the search string in the different electronic databases, an experienced librarian will be involved during this phase of the search.

In addition, to identify any further published or unpublished studies, all the authors of the studies included will be contacted. Reference sections of included studies will be checked. Conference proceedings will be handsearched from inception for abstracts, papers, or posters presented at the following international scientific societies relevant to research on LSS: American Association of Neurological Surgeons, World Federation of Neurosurgical Societies, North American Spine Society, British Association of Spine Surgeons, Spine Society of Europe (Eurospine), AO Spine, American Psychological Association, European Association of Neurosurgical Societies, Society for Health Psychology and European Health Psychology Society. This search will be carried out independently by the two reviewers (AP, VFM) by accessing the websites of these scientific societies. Eligible theses and doctoral dissertations will be searched and identified by the two independent reviewers who will run the same queries using the same keywords on the Open Access 
Theses and Dissertations website. All the searches will be re-run just before the final analyses.

\section{Selection of studies}

Studies will be assessed and screened by two independent reviewers (AP, VFM) in two stages using inclusion/exclusion criteria. During the first stage, studies will be assessed independently by the reviewers with regards to inclusion criteria after reading the title and the abstract. Then, the reviewers will meet to compare their selections. During this stage, only studies on which both reviewers are in complete agreement on exclusion will be excluded. On the contrary, studies will be retained if there is disagreement between the reviewers on inclusion or exclusion. Studies for which there is complete agreement between the reviewers on inclusion will be included. During the final stage, studies will be assessed independently by the two reviewers by assessing the full text of the paper. Potential discrepancies on inclusion or exclusion at this stage and their reasons will be discussed and resolved in a meeting with two other independent reviewers (FF, AC) to obtain an agreed-upon number of included studies. Between-reviewer agreement on inclusion will be calculated by the Kappa index. ${ }^{43}$ During the whole selection process, potential duplicates will be handled and excluded by following the systematic detection heuristic proposed by Wood. ${ }^{44}$

\section{Data extraction}

All information will be extracted from each of the included studies by two independent reviewers (AP, VFM) and inserted into an Excel worksheet after an initial pilot using three included studies. Table 2 provides information on what will be extracted and coded from the primary studies. A third independent reviewer $(\mathrm{FF})$ not involved in the extraction process will check the correctness of the data inserted in the worksheet. After data insertion is completed, potential discrepancies in the data extracted by the two reviewers will be discussed at a meeting between the reviewers who conducted the data extraction and the third independent reviewer.

\section{Measurement of methodological quality of studies}

As in our previous meta-analytical works, ${ }^{11} 45$ the Newcastle-Ottawa Quality Assessment Scale (NOS) will be adopted by two independent meta-analysts to examine the methodological quality of each study ${ }^{46}$ This checklist is based on a maximum score of nine: four points are assigned to inclusion criteria of cases and controls (definition of cases, selection of cases, definition of control subjects, selection of control subjects), two points are assigned to the comparability criteria of cases and control subjects according to study design and statistical analysis (comparability in terms of age and gender), and three points to exposure verification criteria of cases and control subjects (exposure verification, same method of verification, no response point). Studies which obtain a nine score are considered as high quality, those receiving seven or eight as medium quality, and those scoring less than seven as poor quality. A discussion meeting will be planned to resolve eventual disagreement in score attribution between the two meta-analysts.

\section{Meta-analytic procedure}

Summary measures

A random effects meta-analysis will be conducted using the software Comprehensive Meta-Analysis, CMA version $2.00 .{ }^{47}$ For all the analyses, significance will be analysed by quantifying the evidence on a continuous scale. Randomeffects models assume that included studies are drawn from populations of studies that systematically differ from each other. ${ }^{47}$ According to these models, effect sizes extracted from included studies differ because of random error within studies (as in fixed-effect models) and also because of true variation in effect sizes from one study to another. Summary measures will consist of effect-size indexes related to the levels of health-related quality of life in clinical groups as compared with control groups. In addition, effect-size indexes will be calculated for any measures of objective functional impairment (as defined in the 'Eligibility criteria' paragraph).

Effect-size indexes will be calculated using the following formula proposed by Cohen ${ }^{48}: d=\left(\mathrm{M}_{\text {CASE }}-\mathrm{M}_{\text {CONTROL }}\right) /$ $\mathrm{SD}_{\text {COMBINED }}$, where $\mathrm{M}_{\text {CASE }}$ and $\mathrm{M}_{\text {CONTROL }}$ represent the means of the clinical group and control groups, respectively, and $\mathrm{SD}_{\text {COMBINED }}$ is the combined $\mathrm{SD}$. If a study used a measure of health-related quality of life/objective functional impairment that contains subscales (eg, the SF-36), a global effect-size of quality of life and/or functional impairment index will be computed by pooling all the effect-size indexes obtained from the comparison between the clinical group and the controls on each subscale.

The score of each index will be weighted using the following correction formula: $W_{\mathrm{zr}}=1 / \mathrm{SE}^{2}$, where $\mathrm{SE}^{2}$ ir the SE of the effect-size index calculated for each study. Using Cohen's model, effect-size indexes greater than or equal to 0.80 are considered high, indexes in the range of $0.80-0.50$ moderate and indexes in the range of 0.50 0.20 low. Hedges' correction for small sample bias will be applied. ${ }^{49}$

\section{Bias of publication}

In order to investigate whether the effect sizes are subject to a bias of publication, two methods will be adopted: Duval and Tweedie's trim-and-fill technique and a visual examination of the funnel plot. ${ }^{50} \mathrm{~A}$ funnel plot is a scatter plot in which the effect sizes derived from the included papers are plotted on the horizontal axis against an indicator of study precision, the standardised error, on the vertical axis. ${ }^{51}$ In the absence of bias, the graph resembles a symmetrical inverted funnel because the effect sizes calculated from smaller studies scatter more widely at the bottom of the graph, with the spread narrowing as precision increases among larger studies. If there is publication bias because smaller studies showing no significant effect sizes remain unpublished, then the funnel plot results 
Table 2 Information extracted from the primary studies and coding procedure

\begin{tabular}{|c|c|}
\hline Information extracted & Coding \\
\hline Title of the paper & Full title of the paper \\
\hline First author name & First author's last name \\
\hline Publication date & Publication date of the paper \\
\hline Language of the paper & Language in which the paper is written \\
\hline Publication on a peer-review journal & "Yes", "No" \\
\hline Publication type & "Published on a journal", "Conference paper", "Thesis/doctoral dissertation" \\
\hline $\begin{array}{l}\text { Country where the study was } \\
\text { conducted }\end{array}$ & Name of the country \\
\hline Participants' inclusion criteria & Quote the inclusion criteria reported in the study paper \\
\hline Participants' exclusion criteria & Quote the exclusion criteria reported in the study paper \\
\hline Total sample size in the study & Total sample size in the study \\
\hline $\begin{array}{l}\text { Participants with lumbar spinal } \\
\text { stenosis }\end{array}$ & No of clinical participants with lumbar spinal stenosis \\
\hline Control participants & No of control participants \\
\hline Matched controls & $\begin{array}{l}\text { "Yes", "No" } \\
\text { If Yes, specify if match was made on age or gender or both }\end{array}$ \\
\hline Age & $\begin{array}{l}\text { Total study mean age and SD. If the study does not report these data, they will be } \\
\text { requested from the corresponding author. If this is not the case, mean and SD will } \\
\text { be estimated from median and IQRs through the formula proposed by Wan and } \\
\text { colleagues. }{ }^{57} \text { Otherwise, the study will be excluded from the analyses involving data or } \\
\text { age }\end{array}$ \\
\hline Women & Total percentage of women in the study \\
\hline Married/cohabitant patients & Total percentage of married/cohabitant patients \\
\hline Employed patients & Percentage of employed patients \\
\hline Research design & "Cross-sectional case-control”, "Longitudinal” \\
\hline Lumbar spinal stenosis diagnosis & Diagnostic criteria used to establish diagnosis \\
\hline
\end{tabular}

Instrument(s) used to establish lumbar Acronym of the instrument(s)

spinal stenosis diagnosis

Instrument(s) used to assess health- Acronym of the instrument(s)
related quality of life
Type of instrument(s) used to assess "Clinician-administered interview", "Self-report questionnaire"

health-related quality of life

\begin{tabular}{|c|c|}
\hline $\begin{array}{l}\text { Instrument(s) used to evaluate } \\
\text { objective functional impairment }\end{array}$ & Acronym of the instrument(s) \\
\hline Duration of lumbar spinal stenosis & Study mean duration of lumbar spinal stenosis in months \\
\hline Clinical population & "Outpatient", "Inpatient" \\
\hline Strategies used to recruit clinical & Quote the strategies reported in the study paper \\
\hline
\end{tabular}

participants

Strategies used to recruit controls Quote the strategies reported in the study paper

Setting where clinical participants Quote the setting where patients were recruited

were recruited

Comorbidity of depressive disorders Percentage of patients with comorbid depressive disorders in the study according to any version of any international standardised classification systems

Instrument(s) used to assess disability Acronym of the instrument(s)

related to lumbar spinal stenosis

$\begin{array}{ll}\begin{array}{l}\text { Disability related to lumbar spinal } \\ \text { stenosis }\end{array} & \begin{array}{l}\text { Study mean scores on the Swiss Spinal Stenosis Questionnaire and Oxford Spinal } \\ \text { Stenosis Score }\end{array} \\ \begin{array}{l}\text { Instrument(s) used to assess self- } \\ \text { reported pain severity }\end{array} & \text { Acronym of the instrument(s) }\end{array}$


Table 2 Continued

\begin{tabular}{ll}
\hline Information extracted & Coding \\
\hline Self-reported pain severity & Study mean scores on the Visual Analog Scale for pain, Numeric Rating Scale for pain, \\
& McGill Pain Questionnaire
\end{tabular}

asymmetrical. ${ }^{51}$ The aim of the trim-and-fill method is to evaluate the effect of adjustment for bias related to small studies. It removes studies until symmetry in the funnel plot is achieved, recalculating the centre of the funnel before the removed studies are replaced together with their 'missing' mirror-image counterparts. ${ }^{50}$ This procedure will result in a revised summary estimate calculated using all of the original studies, together with the hypothetical 'filled' studies. The new summary estimate (after trim-and-fill) will be reported together with the original estimate in every meta-analysis.

\section{Inconsistency analysis}

To verify heterogeneity in effect sizes, the $I^{2}$ statistic $^{51}$ and the $Q$ index ${ }^{49}$ will be calculated. The $I^{2}$ index is the percentage of variation across studies that is attributable to heterogeneity rather than chance. ${ }^{51} \mathrm{~A}$ value approximating zero suggests homogeneity, whereas values of $25 \%-50 \%, 50 \%-75 \%$ and $75 \%-100 \%$ represent low, moderate and high heterogeneity, respectively. The $Q$ index is calculated by summing the squared deviation of each study's effect estimate from the overall effect estimate, while weighting the contribution of each study by its inverse variance. ${ }^{49}$ In the hypothesis of homogeneity among effect sizes, the $Q$ statistic follows a $\chi^{2}$ distribution with $k-1$ degrees of freedom, $k$ being the number of studies.

\section{Subgroup and moderator analyses}

If significant inconsistency is found, subgroup analyses will be conducted for studies using (a) only clinicianadministered interviews to measure health-related quality of life and (b) self-report questionnaires of health-related quality of life.

Comorbidity of depressive disorders will be investigated as a moderator of the effect sizes through a metaregression. Comorbidity of depressive disorders will be coded as the percentage of patients with comorbid depressive disorders in the study according to the criteria for a major depressive disorder of any standardised international classification systems such as DSM or ICD. If such data are not given in the study paper (ie, the paper does not report on depression, or does not explicitly state the percentage of patients with comorbid depressive disorders), the corresponding author will be contacted to request this information. In this case, the study will be included in the analysis only if the corresponding author is available to provide the necessary data.

Additional moderators will be examined including (a) age coded by the total mean age in the study, (b) female gender coded by the total percentage of females in the study, (c) duration of LSS coded by the mean number of months since the diagnosis in the study, (d) self-reported disability related to LSS coded by the mean scores on the Swiss Spinal Stenosis Questionnaire and Oxford Spinal Stenosis Score $^{52}$ (the moderating effects will be computed separately for each of these two scales if available), (e) pain severity coded by the mean scores on the Visual Analog Scale for pain, Numeric Rating Scale for pain and McGill Pain Questionnaire ${ }^{53}$ (as for disability scores, the moderating effects will be computed separately for each of these scales), and (f) study methodological quality (coded by the scores on the NOS). As for data related to depressive disorders, studies will be included if the paper provides the necessary information or the corresponding author is available to provide it when asked. The relationship between the effect sizes and all these moderators will be investigated by conducting weighted least-squares meta-regression analyses.

If studies with controls without depressive disorders are retrieved, in order to disentangle depression and LSS effects in such studies, the percentage of controls with depressive disorders will be included as moderator in the analysis. This strategy will aim to examine whether the percentage of controls with depression moderates the effect sizes. It can be expected that in studies where the percentage of controls with comorbid depressive disorders is higher, the difference in the quality of life/functional impairment levels between patients and control is lower.

According to Valentine et al's recommendations, ${ }^{54}$ the minimum number of studies for pooling the data and performing effect size calculation will be 2 . Following the guidelines for a continuous study level variable proposed by $\mathrm{Fu}$ et $a l,{ }^{55}$ at least 6 to 10 studies will be necessary to investigate the moderating effects through meta-regression.

\section{Patient and public involvement}

Patients and the public were not involved in the development phase of the research question, of the outcome measures, and of the systematic review and meta-analysis protocol. The study does not involve patient recruitment, and patients were not involved in the conduction of the study. The findings will be disseminated through a publication in a peer-reviewed journal.

\section{Discussion and conclusions}

Health-related quality of life is an impaired psychological outcome among patients with LSS. ${ }^{22}$ Several psychological processes may explain why patients with LSS have poorer quality of life than controls such as worse self-management skills of symptoms, lower engagement 
in daily activities and physical exercise, lower tendency to collaborate with healthcare providers and navigate effectively the healthcare system, and more catastrophic beliefs about pain. ${ }^{24}$ Health-related quality of life is a key outcome in the management of LSS and its assessment during clinical practice may suggest the introduction of psychological interventions directed at promoting psychological resources in the patient with the aim to improve long-term outcome or even enhance recovery. Indeed, cognitive-behavioural patient education has been proven effective for the improvement of both clinical and quality-of-life outcomes in these patients. ${ }^{35} 36$

In the scientific literature, there is no systematic review summarising the evidence of health-related quality of life in LSS. The current paper presents a study protocol of the first systematic review and meta-analysis aimed at summarising health-related quality of life impairment in patients with LSS compared with healthy control groups. Comorbidity of depressive disorders will be investigated as a moderator if significant heterogeneity in the effect sizes is found. Comorbid depressive disorders may be expected to impact on the levels of quality of life because this type of comorbidity is associated with more dysfunctional coping strategies, long-term disability and psychophysiological symptoms interfering with general functioning. ${ }^{31} 32$

Some methodological strengths of the review may be highlighted. First, the review is based on a study selection and a data extraction performed by two independent reviewers; in addition, inter-rater agreement will be evaluated and meeting with other reviewers will be carried out. Comorbid psychiatric disorders will not be excluded as they are quite frequent among patients with LSS; in addition, this allows the review to have sufficient external validity. Any research design will be considered as eligible and this may allow additional eligible data to be located and included even if the focus of the paper is not the comparison of health-related quality of life between patients and controls. Moreover, the search strategy is based on the identification of published and unpublished studies. Lastly, another strength is the evaluation of the study's methodological quality through a specific tool.

The review may have important clinical implications: for example, it can highlight the importance of focusing the assessment also on quality of life in patients with LSS to improve prognosis and treatment response because some psychological interventions have been proven effective for this condition. ${ }^{35}$

Since one of the most strongly affected areas of daily life in LSS is the ability to perform work-related activity, a summary of the evidence about depression and impaired quality of life in this condition can suggest that occupational health professionals should assess these outcomes during occupational medicine practice with the aim to improve work-related health and functioning. For example, well informed physician-patient communication in consultations on back pain may prevent deterioration of the LSS and improve patients' perceived health status. ${ }^{56}$ Our paper may suggest that occupational medicine practice may be improved by the use of instruments aimed at a timely detection of depression and perceived health status impairment.

Finally, potential limitations of the review regard a small number of studies in the literature and the heterogeneity of the studies in terms of the instruments used to assess health-related quality of life and the difference in the definitions used to conceptualise this construct. Another potential problem is that some studies will not report on the data necessary to code the moderators (eg, they will not explicitly state the percentage of depressive disorders) or the authors are not available to provide them.

In conclusion, this is a protocol of the first systematic review of health-related quality of life in patients with LSS. A summary of the evidence on this topic may support clinical practice highlighting the importance of the assessment of quality of life and suggesting the use of psychological interventions dedicated to this outcome with the aim of improving patients' quality of life.

Contributors FF conceived and designed the study, wrote the first draft of the paper and reviewed the final draft. AC conceived and designed the study, critically reviewed the first draft of the paper and reviewed the final draft. RG critically reviewed the last draft of the paper. GG critically reviewed the last draft of the paper. VFM conceived and designed the study, and critically reviewed the first draft of the paper. AP conceived and designed the study, wrote the first draft of the paper and reviewed the final draft. All the authors approved the final version of the manuscript.

Funding The authors have not declared a specific grant for this research from any funding agency in the public, commercial or not-for-profit sectors.

Competing interests None declared.

Patient consent for publication Not required.

Provenance and peer review Not commissioned; externally peer reviewed.

Open access This is an open access article distributed in accordance with the Creative Commons Attribution Non Commercial (CC BY-NC 4.0) license, which permits others to distribute, remix, adapt, build upon this work non-commercially, and license their derivative works on different terms, provided the original work is properly cited, appropriate credit is given, any changes made indicated, and the use is non-commercial. See: http://creativecommons.org/licenses/by-nc/4.0/.

\section{ORCID iDs}

Vitaliano Francesco Muzii http://orcid.org/0000-0002-2346-8728

Andrea Pozza http://orcid.org/0000-0002-6634-6106

\section{REFERENCES}

1 DuBois CM, Beach SR, Kashdan TB, et al. Positive psychological attributes and cardiac outcomes: associations, mechanisms, and interventions. Psychosomatics 2012;53:303-18.

2 Sheldon KM, King L. Why positive psychology is necessary. Am Psychol 2001;56:216-7.

3 Bullinger M, Anderson R, Cella D, et al. Developing and evaluating cross-cultural instruments from minimum requirements to optimal models. Quality of Life Research 1993;2:451-9.

4 Castro EM, Van Regenmortel T, Vanhaecht K, et al. Patient empowerment, patient participation and patient-centeredness in hospital care: a concept analysis based on a literature review. Patient Educ Couns 2016;99:1923-39.

5 Coluccia A, Fagiolini A, Ferretti F, et al. Obsessive-compulsive disorder and quality of life outcomes: protocol for a systematic review and meta-analysis of cross-sectional case-control studies. Epidemiology, Biostatistics and Public Health 2015;12:e11037.

6 Coluccia A, Ferretti F, Fagiolini A, et al. Quality of life in children and adolescents with obsessive-compulsive disorder: a systematic review and meta-analysis. Neuropsychiatr Dis Treat 2017;13:597-608. 
7 Pozza A, Lochner C, Ferretti F, et al. Does higher severity really correlate with a worse quality of life in obsessive-compulsive disorder? A meta-regression. Neuropsychiatr Dis Treat 2018;14:1013-23.

8 Johansson P, Dahlström U, Broström A. Factors and interventions influencing health-related quality of life in patients with heart failure: a review of the literature. Eur J Cardiovasc Nurs 2006;5:5-15.

9 Leadley RM, Armstrong N, Lee YC, et al. Chronic diseases in the European Union: the prevalence and health cost implications of chronic pain. J Pain Palliat Care Pharmacother 2012;26:310-25.

10 World Health Organization. Preamble to the constitution of the World Health Organization. in official records of the World Health Organization, no. 2 (P. 100). Geneva: author, 1948. Available: http:// whqlibdoc.who.int/hist/official_records/2e.pdf

11 Porcelli B, Pozza A, Bizzaro N et al. Association between stressful life events and autoimmune diseases: a systematic review and meta-analysis of retrospective case-control studies. Autoimmun Rev 2016;15:325-34.

12 Resnick DK, Watters WC, Sharan A, et al. Guideline update for the performance of fusion procedures for degenerative disease of the lumbar spine. Part 9: lumbar fusion for stenosis with spondylolisthesis. J Neurosurg 2014;21:54-61.

13 Lurie J, Tomkins-Lane C. Management of lumbar spinal stenosis. BMJ 2016;4.

14 Konno S, Hayashino Y, Fukuhara S, et al. Development of a clinical diagnosis support tool to identify patients with lumbar spinal stenosis. Eur Spine J 2007;16:1951-7.

15 Spengler DM. Degenerative stenosis of the lumbar spine. J Bone \& Joint Surgery 1987;69:305-8.

16 Kreiner DS, Shaffer WO, Baisden JL, et al. An evidence-based clinical guideline for the diagnosis and treatment of degenerative lumbar spinal stenosis (update). Spine Journal 2013;13:734-43.

17 Cowley P. Neuroimaging of spinal canal stenosis. Magn Reson Imaging Clin N Am 2016;24:523-39.

18 Katz JN, Dalgas M, Stucki G, et al. Degenerative lumbar spinal stenosis diagnostic value of the history and physical examination. Arthritis \& Rheumatism 1995;38:1236-41.

19 Katz JN, Harris MB. Clinical practice. lumbar spinal stenosis. N Engl J Med 2008;358:818-25.

20 Rainville J, Childs LA, Peña EB, et al. Quantification of walking ability in subjects with neurogenic claudication from lumbar spinal stenosis - a comparative study. Spine J 2012;12:101-9.

21 Kent DL, Haynor DR, Larson EB, et al. Diagnosis of lumbar spinal stenosis in adults: a metaanalysis of the accuracy of CT, MR, and myelography. AJR Am J Roentgenol 1992;158:1135-44.

22 Otani K, Kikuchi S, Yabuki S, et al. Lumbar spinal stenosis has a negative impact on quality of life compared with other comorbidities: an epidemiological cross-sectional study of 1862 communitydwelling individuals. Scientific World J 2013;2013:1-9.

23 Saban KL, Penckofer SM, Androwich I, et al. Health-related quality of life of patients following selected types of lumbar spinal surgery: a pilot study. Health Qual Life Outcomes 2007;5:71.

24 Skolasky RL, Mackenzie EJ, Riley LH, et al. Psychometric properties of the patient activation measure among individuals presenting for elective lumbar spine surgery. Qual Life Res 2009;18:1357-66.

25 Von Korff M, Gruman J, Schaefer J. Collaborative management of chronic illness. Ann Intern Med 1997;127:1097-102.

26 Sekiguchi M, Yonemoto K, Kakuma T, et al. Relationship between lumbar spinal stenosis and psychosocial factors: a multicenter crosssectional study (DISTO project). Eur Spine J 2015;24:2288-94.

27 Levy HI, Hanscom B, Boden SD. Three-question depression screener used for lumbar disc herniations and spinal stenosis. Spine 2002;27:1232-6.

28 Sinikallio S, Aalto T, Airaksinen O, et al. Depression and associated factors in patients with lumbar spinal stenosis. Disabil Rehabil 2006;28:415-22.

29 Coronado RA, George SZ, Devin CJ, et al. Pain sensitivity and pain catastrophizing are associated with persistent pain and disability after lumbar spine surgery. Arch Phys Med Rehabil 2015:96:1763-70.

30 McKillop AB, Carroll LJ, Battié MC. Depression as a prognostic factor of lumbar spinal stenosis: a systematic review. Spine 2014;14:837-46.

31 Sinikallio S, Aalto T, Airaksinen O, et al. Depression is associated with a poorer outcome of lumbar spinal stenosis surgery: a two-year prospective follow-up study. Spine 2011;36:677-82.

32 Sinikallio S, Aalto T, Koivumaa-Honkanen $\mathrm{H}$, et al. Life dissatisfaction is associated with a poorer surgery outcome and depression among lumbar spinal stenosis patients: a 2-year prospective study. Eur Spine J 2009;18:1187-93.
33 Stienen MN, Smoll NR, Joswig $\mathrm{H}$, et al. Influence of the mental health status on a new measure of objective functional impairment in lumbar degenerative disc disease. Spine J 2017;17:807-13.

34 Stienen MN, Ho AL, Staartjes VE, et al. Objective measures of functional impairment for degenerative diseases of the lumbar spine: a systematic review of the literature. Spine J 2019;19:1276-93.

35 Archer KR, Devin CJ, Vanston SW, et al Cognitive-behavioral-based physical therapy for patients with chronic pain undergoing lumbar spine surgery: a randomized controlled trial. J Pain 2016;17:76-89.

36 Rolving N, Sogaard R, Nielsen CV, et al. Preoperative cognitivebehavioral patient education versus standard care for lumbar spinal fusion patients: economic evaluation alongside a randomized controlled trial. Spine 2016;41:18-25.

37 Shamseer L, Moher D, Clarke M, et al. Preferred reporting items for systematic review and meta-analysis protocols (PRISMA-P) 2015: elaboration and explanation. BMJ 2015;349:97647.

38 Birkmeyer NJO, Weinstein JN, Tosteson ANA, et al. Design of the Spine Patient outcomes Research Trial (SPORT). Spine 2002;27:1361-72.

39 Ware JE, Sherbourne CD. The MOS 36-Item short-form health survey (SF-36). Med Care 1992;30:473-83.

40 American Psychiatric Association. Diagnostic and statistical manual of mental disorders. 4th ed. Washington, DC: American Psychiatric Association, 2000.

41 American Psychiatric Association. Diagnostic and statistical manual of mental disorders. 5th ed. Washington, DC: American Psychiatric Association, 2013.

42 Diebo BG, Lavian JD, Murray DP, et al. The impact of comorbid mental health disorders on complications following adult spinal deformity surgery with minimum 2-year surveillance. Spine 2018;43:1176-83.

43 Cohen J. A coefficient of agreement for nominal scales. Educ Psychol Meas 1960;20:37-46.

44 Wood JA. Methodology for dealing with duplicate study effects in a meta-analysis. Organ Res Methods 2008;11:79-95.

45 Pozza A, Coluccia A, Kato T, et al. The 'Hikikomori' syndrome: worldwide prevalence and co-occurring major psychiatric disorders: a systematic review and meta-analysis protocol. BMJ Open 2019;9:e025213.

46 Wells GA, Shea B, O'Connell D, et al. The Newcastle-Ottawa scale (NOS) for assessing the quality of non-randomised studies in metaanalyses, 2000. Available: http://www. ohri.ca/programs/clinical epidemiology/oxford.asp

47 Borenstein M, Hedges LV, Higgins JPT, et al. Introduction to metaanalysis. Chichester: John Wiley \& Sons, 2009.

48 Cohen J. Statistical power analysis for the behavioural sciences. 2nd ed. Hillsdale, NK: Erlbaum Ed, 1988.

49 Hedges LV. Estimation of effect size from a series of independent experiments. Psychol Bull 1982;92:490-9.

50 Duval S, Tweedie R. Trim and fill: a simple funnel-plot-based method of testing and adjusting for publication bias in meta-analysis. Biometrics 2000;56:455-63.

51 Higgins JPT, Thompson SG, Deeks JJ. Measuring inconsistency in meta-analyses. BMJ 2003;327:557-60.

52 Pratt RK, Fairbank JCT, Virr A. The reliability of the Shuttle Walking Test, the Swiss Spinal Stenosis Questionnaire, the Oxford Spinal Stenosis Score, and the Oswestry Disability Index in the assessment of patients with lumbar spinal stenosis. Spine 2002;27:84-91.

53 Hawker GA, Mian S, Kendzerska T, et al. Measures of adult pain: Visual Analog Scale for Pain (VAS Pain), Numeric Rating Scale for Pain (NRS Pain), McGill Pain Questionnaire (MPQ), Short-Form McGill Pain Questionnaire (SF-MPQ), Chronic Pain Grade Scale (CPGS), Short Form-36 Bodily Pain Scale (SF-36 BPS), and Measure of Intermittent and Constant Osteoarthritis Pain (ICOAP). Arthritis Care Res 2011;63:S240-52.

54 Valentine JC, Pigott TD, Rothstein HR. How many studies do you need? A primer on statistical power for meta-analysis. Journal of Educational and Behavioral Statistics 2010;35:215-47.

55 Fu R, Gartlehner G, Grant M, et al. Conducting quantitative synthesis when comparing medical interventions: $A H R Q$ and the Effective Health Care Program. J Clin Epidemiol 2011;64:1187-97.

56 Voigt-Radloff S, Schöpf AC, Boeker M, et al. Well informed physician-patient communication in consultations on back painstudy protocol of the cluster randomized GAP trial. BMC Fam Pract 2019;20:33

57 Wan X, Wang W, Liu J, et al. Estimating the sample mean and standard deviation from the sample size, median, range and/or interquartile range. BMC Med Res Methodol 2014;14:135. 